\title{
Report: A patient with melioidosis presenting with fever, dysuria and hip pain: A diagnostic dilemma
}

\author{
GCS Gunasekera, SK Jayatilleke, LN Seneviratne, SASP Subasinghe, CI Kannangara,HL \\ Thushari
}

\section{Introduction}

Melioidosis, caused by Burkholderiapseudomallei, is endemic in South/Southeast Asia. It is reemerging in Sri Lanka, probably due to increased awareness among microbiologists and clinicians. However, being a diagnostic dilemma due to its varied presentations, this great mimicker requires a high degree of clinical suspicion for early diagnosis and optimal antimicrobials to reduce morbidity/mortality.

\section{Case report}

An elderly diabetic female presented with fever for 1 month, dysuria and left-sided hip pain. WBC and UFR were normal but CRP was high. Urine culture was negative but blood culture became positive, resembling a mixed growth. Upon standing, the culture manifested as a single type of wrinkled colonies with typical features of B. pseudomallei. Suggestive antibiogram and non-identification by Vitek ${ }^{\circledR}$ led to reference laboratory identification. Antibodies against B. pseudomallei were also high (>10240).

In spite of worsening pain, X-Ray/USS revealed a normal hip joint. Double dose meropenem was started. MRI revealed joint effusion indicating septic arthritis. CT-KUB/CECT-abdomen showed bilateral renal cysts and a peri-nephritic collection. Meropenem dose was lowered after excluding CNS involvement. Oral cotrimoxazole was added. At the time of submission, the patient is on 20 days of induction therapy, is awaiting repeat imaging and is clinically better. Eradication therapy with oral cotrimoxazole/doxycycline for $\geq 3$ months is planned.

\section{Discussion and Conclusion}

B. pseudomallei is a soil-dwelling bacterium. Inhalation or direct inoculation through damaged skin are the routes of transmission. The patient was exposed to her pet dog that brought soil into the house after rolling outside. Septic arthritis is a well-known manifestation of melioidosis. Renal cysts in this patient may represent renal involvement. In this instance, if the blood culture result was disregarded as mixed due to contamination and if the patient's complaint was unheeded due to initially normal investigations, the diagnosis would have been missed. A high index of clinical suspicion is needed for diagnosis and treatment of melioidosis.

Department of Microbiology, Sri Jayewardenepura General Hospital, Nugegoda, Sri Lanka.

Address for correspondence: Dr ChathuriGunasekara, Department of Microbiology, Sri Jayewardenepura General Hospital, Nugegoda, Sri Lanka +94 773595610 Email: chathurigcs@yahoo.com 1 https://orcid.org/0000-00025141-6319 